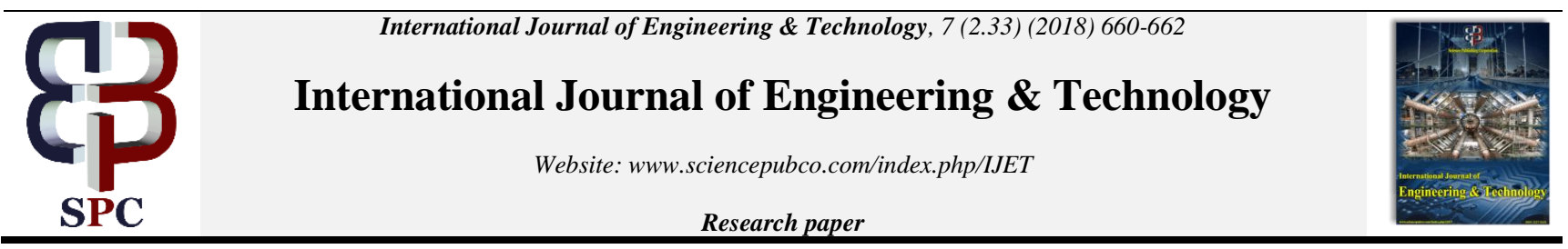

\title{
Mobility models for an asynchronous wireless sensor multimedia network
}

\author{
Praveen Kumar $\mathrm{D}^{1}{ }^{*}$, Dr. Jerritta $\mathrm{S}^{2}$ \\ ${ }^{1}$ Research Scholar, Department of ECE, VISTAS, Chennai, India \\ ${ }^{2}$ Associate Professor, Associate Professor, Department of ECE, VISTAS, Chennai, India \\ *Corresponding author E-mail: damodaranpraveenkumar@gmail.com
}

\begin{abstract}
In Wireless Sensor Multimedia Networks, the movement models and tracking mechanisms have important roles on Quality of data collection and network delay. The various movement models in a hierarchically connected network entities are discussed in a Wild life Habitat moni-toring scenario. The quality of routing decisions depends on how accurately we can predict the mobility pattern of nodes. A simulator test-ing the different mobility models is discussed in this paper.
\end{abstract}

Keywords: Base Station (BS); Cluster Heads (CH); Entity of Interest (EOI); Sensor Nodes (SN); Wireless Multimedia Sensor Network (WSMN).

\section{Introduction}

In a Wireless Sensor Network (WSN), the basic movement models used in the communication between wireless nodes is considered here[7]. In physical layer the nodes communicates in bits. The stream of bits are coded for achieving coding efficiency. But higher layers are responsible for mobility management and routing decisions. Experimenting mobility models in a real world set up may not be feasible. This is the motivation behind a WSN simulator where different Mobility management strategies are explored. One of the active research area in WSN is that pertaining to Wireless Multimedia Sensor Networks (WMSN) dealing with high data requirement. Wireless Sensor Network Implementation moves toward multimedia applications which necessitate more energy consumption every other day .Special sensor networks designed primarily for the delivery of multimedia content are called Wireless Multimedia Sensor Networks. Since a huge amount of data such as : (i) Audio, (ii) Still video and (iii) Streaming Video are to be handled and to be processed on a near real time basis, these application are bandwidth hungry to meet the required quality of Service. Surveillance applications domain is among the popular implementation areas of Wireless Multimedia Sensor Networks [1-6]. Different sensor devices of various data capability are to be used in such a monitoring application.

\section{Habitat monitoring scenario}

For this application scenario, the simulation depends on a hierarchical structure where the data collecting points are moving Wireless Sensor nodes which are available in a pre-defined geographical region of Interest (RoI) [3-9]. In a given RoI several nodes will be available and each will be having an entity of Interest (EoI) EoI is a subset of RoI. The sensor nodes are reporting to the manager node known as Cluster Head $(\mathrm{CH})$ and the manager node send information to the gateway sink, known as Base Station
(BS).The BS acts as a gateway to the internet cloud or any other transit network.

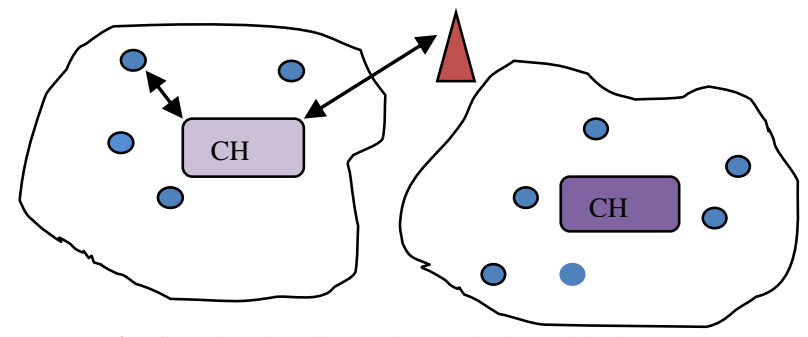

Fig. 1: Roi and Eoi in a Clustered Hierarchical WMSN.

\section{Mobility and tracking for WMSN}

The mobility of sensor nodes is the vital driving factor for Detection and tracking of targets in this case Wild-life Habitat monitoring. Detection of edges or of counters in the level of continuous physical action and to obtain an estimate of the physical field. Position information is heavily used [10].

\subsection{Detection}

A node can autonomously detect the target's presence by comparing its own sensor inputs against some application specific threshold values.

\subsection{Target Localisation}

Multiple sensor inputs are correlated for a specific target detection.

\subsection{Decision}

Classification of Time series inputs can be done at a hierarchically higher node in this case at Cluster Head level. 


\subsection{Tracking}

All nodes sensing the target report time stamped readings to the sink node which assimilates the data to obtain desired decision. A manager node, here the cluster head $(\mathrm{CH})$, in the vicinity of the target collects local sensor readings and estimates the target position. By knowing the current position as well as past positions and their time stamps, the $\mathrm{CH}$ can estimate the speed and heading of the target or object .As the object moves away, the manager node $(\mathrm{CH})$ hands off the whole target State to a new Cluster Head. The target state includes (i) history of positions including the current one (ii) speed and (iii) the heading information. This new node is chosen such that its location is close to the projected target position at the time of handoff; the choice is thus geography based. In this manner the estimated state follows the target in space and time. Additionally the manager nodes send target position reports to the sink from time to time.

\section{A movement models}

Mobility pattern of the sensor nodes are termed as Movement models. In a classic way-point model the nodes chooses a destination randomly and heads towards it. While reaching the destination, the node again selects another random destination and the process continues. Since we uses the movement of sensor nodes through a geographical path, we have to consider the characteristics of the paths under consideration. For example, the path type and the type of moving infrastructure for the sensor nodes. The drones in which sensors are fitted will be having different path type rather than a terrestrial vehicular infrastructure.

The three different type of movement models for this habitat monitoring simulator is explained here. (i) Stationary Movement (ii) Simple Random movement (iii) Path-Type Movement.

\subsection{Stationary movement}

Stationary movement refers to fixed nodes in the network. Static nodes are sink nodes in this simulation and termed as the cluster heads (CHs)and the next higher level entity known as Base Station (BS) which acts as a gateway to the external legacy network. The BS nodes are also defined as stationary. These Gateway nodes are the interface to the external cloud. This gateway node collects report from the manager nodes ie Cluster Heads for object identification, monitoring and tracking.

\subsection{Simple random movement}

It is the simplest mobility model for the wireless sensor nodes. Simple random mobility model is an adoption of random waypoint model with respect to a terrestrial path or an air borne path. Unlike the stationary movement, the node move from one vertex to the adjacent vertex through the edge connecting them. A moving node incidentally at a vertex randomly chooses a vertex from its adjacent vertices, excluding the adjacent vertex that it comes from and moves heads toward the selected vertex through the edge connecting those two vertices. If the vertex is an end point of a terrestrial path, the node traces back to its previous vertex[8].

This model is achieved by inheriting the properties of the stationary node and further defining the following additional attributes

- The path_id of a way where the objects locates.

- The speed of the moving sensor node in $\mathrm{Km} / \mathrm{hr}$.

- The list of path_ids of the ways the node already visited.

- The time_flight of the object in seconds

- mvmt_points: A list to keep the movement points along the trajectory of the node path.

- mvmt_pt_index: Represents the index of the list mvmt_points,which represents the current position of the node.
- Apart from the mobility specific attributes three other functions are also defined to handle the movement of sensor nodes.

\subsection{Addition of new movement models}

The object oriented approach of the WMSN simulator provide an easy way to define custom mobility models. The class SimpleRandomMovement provides the basic movement for the objects and Path-type movement provides a realistic movement of sensor nodes attached to different means of infrastructure for movement. Thus we can distinguish terrestrial sensor nodes and air-borne drones,

Steps involved in adding a new Mobility model

The existing mobility model is to be imported.

The new file should contain a class with the new model definition Re-define initial_node and compute_next_node functions for the new model.

Editing the creat_mvmt_object function in initialization module by inserting an elif statement.

Assign the movement model for a group of mobile nodes using the group parameter Movement in settings file simltr.config ,the simulator configuration file.

Inheritance in object oriented approach makes us to redefine only the changes from existing movement model. Since the computation of next_node determines the movement, the redefinition of the function compute_next_node will be the core part of the new mobility model.

\section{Routing strategy}

Similar to the objective of tracking is to detect the target entering inside a RoI of the WMSN, the objective of routing is to decide whether data is to handed over when two nodes are coming in contact with each other. As we know that in a hierarchical distribution of Network entities, Sensor nodes need not communicate each other. The nodes are destined to communicate with its manager nodes, namely Cluster Heads. Cluster Heads may or may not be talking each other depending on the configuration definition. The next level of entity is the base station which acts as a Gateway to the external network

\section{Routing strategy}

The simulation environment is selected as a geographical area (RoI) which is deadly affected by intrusion of Wild-life viz elephants and leopards from nearby thick forest land and acts as a threat to human lives and destruction of crops. In densely populated area human dwelling may be very close to declared forest areas. Such areas are vulnerable for intrusion of wild animals and a Early warning system will be a boon to such human settlement.

Simulator Architecture: The WMSN simulator is divided into modules as follows.

1) Settings: Module to set the simulation environment.

2) XML Parser: Module to parse the map file (Which is XML format) and convert it into simulation variables.

3) Initialisation: Initialises the Simulator environment using the map data and the users' settings file.

4) Movement Models: Handles different mobility types for the different type of entities.

5) Routing: Related to data transference between network entities.

GUI and Reports: To set a display environment for running the simulation and report generation

GUI: Computations required for GUI are implemented as functions.

Python networkx library: networkx library of Python is used for creating graph . 
Cartesian co-ordinate to geographical co ordinate conversion by knowing the upper and lower bounds of the geographical map is carried out.

Event Logs: Creating sensible log reports is also a part of the simulation.

Events reports: Event refers to occurrence of action. In our scenario even is a signaling from sensor node to next higher level nodes. Classes and functions for describing the event are event_id, event_time, event_counter, event_expired status as bolean etc. Three reports are generated in the GUI report pane. The following are three sub windows visible in the report pane 1. Statistics related to Sensor Node events 2.Proximity statistics and 3. Signalling between Nodes [4].

The Software tool for Simulation

Ubuntu 16.04 LTS is the operating system used. Python 2.7 was used as the programming environment.

Each Component of the WSN simulation is implemented as class. Network elements either stationary or mobile exploit the characteristics of object oriented programming (OOPS) concepts. Stationary nodes are implemented as classes with common attributes such as unique _id, position in the geographical area and buffer storage for data. Inheriting the properties of a stationary object and with additional feature for mobility mobile nodes based class is realized .New mobility model(s) can be created from basic movement models and by adding new features. Since Python supports OOPS the simulator environment is flexible and enhancements are an easy task.

Modules and Functions: Seven important modules are part of the WSN Simulator.. Settings module define environment for execution. The simulator consists of a geographical area. And movement objects both stationary and mobile. The geographical area is specified as a map represented using XML. The map is exported from www.openstreetmap.org in .osm format [5]. A XML parser module is used for the conversion of .osm format to $\mathrm{xml}$ format. In general all the environment parameters are specified in a simulation configuration file.

Mobility models are handled in mobility module. Routing module refers simple data transfer rule.GUI and reports are also the integral parts of the simulator.

GUI is created using Python Tkinter library. Movement reports and event reports are generated using functions in report module.

Table 1: Different Modules in Wmsn Simulator

\begin{tabular}{|c|c|c|}
\hline Sl_No & \multicolumn{2}{|c|}{ Wsn Simulator Modules } \\
\hline & Module & Functionality \\
\hline 01 & Settings & To set the simulation Environment \\
\hline 02 & XML Parser & $\begin{array}{l}\text { To convert map file in to simulation varia- } \\
\text { bles }\end{array}$ \\
\hline 03 & Initialization & Initialises the simulator environment \\
\hline 04 & Mobility Model & Handles different movement models \\
\hline 05 & Routing & Defines routing protocols for the objects \\
\hline 06 & GUI & The Graphical User Interface \\
\hline 07 & Reports & Creates simulation related logs and reports \\
\hline
\end{tabular}

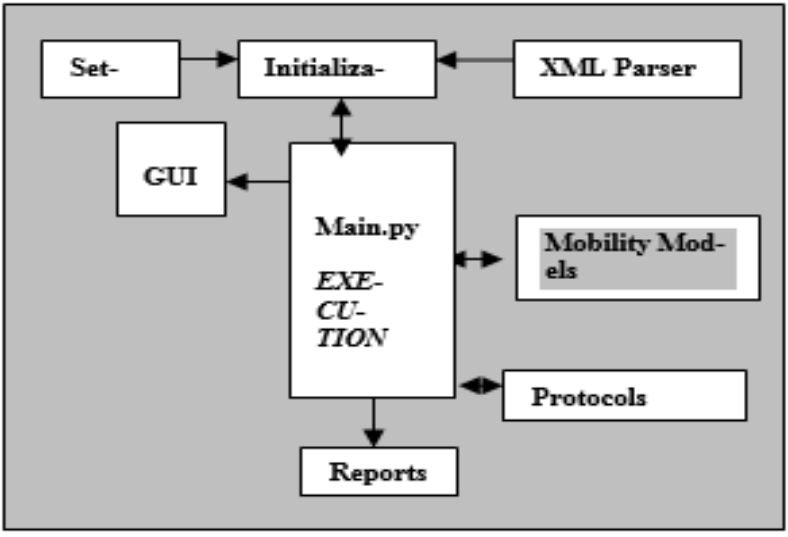

Fig. 2: The WMSN Mobility Simulation Structure.

\section{Conclusion and future work}

This paper addresses the mobility management in Wireless Sensor Multimedia Networks (WMSN) in a Wild-life Habitat monitoring support case [2]. Simulator for evaluating different Movement models for every network entities are explored. The statistical reports generated are related to event generation proximity statistics of moving nodes and thereby the effectiveness of Movement model can be vetted. Suitable routing protocols for a hierarchical assignment is to be tested and the further physical layer communication phenomena like rate matching is to be explored. The features of Mobile Sink based WMSN is the focus of future simulations [1].

\section{Acknowledgement}

We acknowledge Dr.Rajendran V., HOD, Dept. Of ECE, VELS University, Chennai for his valuable suggestion and guidance in selecting the tool for this simulation

\section{References}

[1] Gaolei Li, Mianxiong Dong, Kaoru Ota, Jun Wu, Jianhua Li,Tianpeng Ye, "Towards QoE Named Content-Centric Wireless Multimedia Sensor Networks with Mobile Sinks", Shanghai Jiao Tong University, Shanghai, China., IEEE 2017.

[2] Ruchi Mittal and M.P.S Bhatia, "Wireless Sensor Networks for monitoring the environment activities, IEEE, 2010.

[3] Naregalkar Akshay M. Praveen Kumar and B. Harish, "An Efficient Approach for Sensor Deployments in Wireless Sensor Network," IEEE, 2010.

[4] Kelvin fall, Delay Tolerant Networking, 3rd ed., vol. 2. Oxford: Clarendon, 1892, pp.68-73.

[5] "Data Fusion and Processing in Wireless Multimedia Sensor Networks: An Analysis for Surveillance Applications.2014 IEEE 22 $2^{\text {nd }}$ Signal Processing and Communications Applications Conference (SIU 2014).

[6] F.Nadeem, E.Leitgeb,M.S.Awan "Comparing the lifetime of Terrestrial Wireless Sensor Networks by Employing Hybrid FSO/RF and only RF access Networks," IEEE 2009.

[7] Xiaofeng Han, Xiang Cao,Error L.Lyod,Chien -Chung Shen "Fault-tolerant Relay Node placement in Heterogenous Wireless Sensor Networks", IEEE,2007.

[8] Shangwei Duan, and Xiaobu Yuan, "Exploring Hierarchy Architecture for Wireless Sensor Management," IEEE, 2006.

[9] Holdger Karl, Andreas Willing, "Protocols and Architectures for Wireless Sensor Networks" John Wiley \& Sons, Reprint 2016, pp.425-435. 